\title{
A chaperone network controls the heat shock response in $E$. coli
}

\author{
Eric Guisbert, ${ }^{1}$ Christophe Herman, ${ }^{2,4,5}$ Chi Zen Lu, ${ }^{2}$ and Carol A. Gross ${ }^{2,3,6}$ \\ Departments of ${ }^{1}$ Biochemistry and Biophysics, ${ }^{2}$ Microbiology and Immunology, and ${ }^{3}$ Stomatology, University of California, \\ San Francisco, San Francisco, California 94143, USA; ${ }^{4}$ Department of Molecular and Human Genetics, Baylor College of \\ Medicine, Houston, Texas 77030, USA
}

\begin{abstract}
The heat shock response controls levels of chaperones and proteases to ensure a proper cellular environment for protein folding. In Escherichia coli, this response is mediated by the bacterial-specific transcription factor, $\sigma^{32}$. The DnaK chaperone machine regulates both the amount and activity of $\sigma^{32}$, thereby coupling $\sigma^{32}$ function to the cellular protein folding state. In this manuscript, we analyze the ability of other major chaperones in $E$. coli to regulate $\sigma^{32}$, and we demonstrate that the GroEL/S chaperonin is an additional regulator of $\sigma^{32}$. We show that increasing the level of GroEL/S leads to a decrease in $\sigma^{32}$ activity in vivo and this effect can be eliminated by co-overexpression of a GroEL/S-specific substrate. We also show that depletion of GroEL/S in vivo leads to up-regulation of $\sigma^{32}$ by increasing the level of $\sigma^{32}$. In addition, we show that changing the levels of GroEL/S during stress conditions leads to measurable changes in the heat shock response. Using purified proteins, we show that that GroEL binds to $\sigma^{32}$ and decreases $\sigma^{32}$-dependent transcription in vitro, suggesting that this regulation is direct. We discuss why using a chaperone network to regulate $\sigma^{32}$ results in a more sensitive and accurate detection of the protein folding environment.
\end{abstract}

[Keywords: sigma32 $\left(\sigma^{32}\right)$; GroEL; chaperone; heat shock response; sigma factor ( $\sigma$ factor); DnaK]

Received May 7, 2004; revised version accepted September 9, 2004.

Cellular survival depends on maintaining an appropriate environment for protein folding. Chaperones, which assist protein folding, and proteases, which remove misfolded proteins, are among the cellular factors that influence protein folding in vivo (Horwich et al. 1999; Bukau et al. 2000). Environmental factors, including temperature and organic solvents, also influence the internal folding milieu (Hartl 1996). In order to maintain homeostasis for protein folding, cells tightly regulate the expression of chaperones and proteases to compensate for environmental perturbations (Morimoto 1998). This response, called the "heat shock response" because it was identified in relation to heat stress, leads to the induction of the almost universally conserved "heat shock genes", which encode chaperones, proteases, and other stress-related proteins (Herman and Gross 2000). Heat shock proteins are not only important during stress conditions; many are among the most abundant proteins in the cell in all conditions because they have a general role in protein folding (Nollen and Morimoto 2002).

A complex control system regulates expression of heat shock genes, ensuring that they can respond even to small changes in intracellular folding. In Escherichia

Corresponding authors.

${ }^{5}$ E-MAIL herman@bcm.tmc.edu; FAX (713) 798-2126.

${ }^{6}$ E-MAIL cgross@cgl.ucsf.edu; FAX (415) 514-4080.

Article and publication are at http://www.genesdev.org/cgi/doi/10.1101/ gad.1219204. coli, regulation of heat shock genes is mediated by rpoH, which encodes the $\sigma^{32}$ transcription factor /Connolly et al. 1999; Arsene et al. 2000). $\sigma$ factors are bacterialspecific initiation factors that recruit RNA polymerase to particular classes of promoters (Gruber and Gross 2003). $\sigma^{32}$ is regulated at multiple levels that use inputs both directly from some stresses and also from the protein folding state of the cell. Increases in temperature have been shown to directly increase translation of $r p o H$ mRNA by destabilizing an RNA structural element overlapping the translation start point (Morita et al. $1999 a, b)$. The protein folding state of the cell regulates both the degradation and activity of $\sigma^{32}$. In response to an increased need for protein folding agents (e.g., immediately after temperature upshift), $\sigma^{32}$ is transiently stabilized (Straus et al. 1987). In response to decreased need for protein folding agents (e.g., during the recovery phase following temperature upshift or immediately after temperature downshift), the activity of $\sigma^{32}$ decreases (Straus et al. 1989). Additionally, cells use these feedback systems to constantly monitor their folding status during growth under steady-state conditions so that the cellular folding environment remains optimal.

The DnaK chaperone machine, consisting of DnaK (Hsp70 homolog), DnaJ (Hsp40 homolog), and GrpE (nucleotide exchange factor), is implicated in regulation of $\sigma^{32}$ activity and $\sigma^{32}$ degradation (Tilly et al. 1983, 1989). Mutations in dnaK, dnaJ, and grpE all induce the heat shock response through increases in $\sigma^{32}$ stability 
and activity (Straus et al. 1990). Also, DnaK overexpression and depletion in vivo leads to changes in $\sigma^{32}$ activity and degradation (Tomoyasu et al. 1998). Activity control has been replicated in vitro, as DnaK binds directly to $\sigma^{32}$ and inhibits $\sigma^{32}$-dependent transcription in vitro /Gamer et al. 1992, 1996; Liberek et al. 1992). However, the role of DnaK in the regulation of $\sigma^{32}$ degradation is less clear because it has not been possible to replicate this control in vitro.

The current model for regulation of $\sigma^{32}$ by the DnaK chaperone machine has been referred to as the "unfolded protein titration model" (Straus et al. 1990; Craig and Gross 1991; Bukau 1993). In this model, unfolded proteins and $\sigma^{32}$ compete for binding to DnaK, with the DnaK $-\sigma^{32}$ complex inactive in transcription. In addition, DnaK binding to $\sigma^{32}$ facilitates $\sigma^{32}$ degradation in an unknown way. When unfolded proteins are low relative to DnaK, the inactive DnaK- $\sigma^{32}$ complex predominates, $\sigma^{32}$ is rapidly degraded, and heat shock gene expression is low. However, when unfolded proteins are high relative to DnaK, DnaK is titrated away from $\sigma^{32}$; the active, stable, chaperone-free state of $\sigma^{32}$ predominates; and heat shock genes are induced.

A chaperone network controls protein folding in the cell (Buchberger et al. 1996); therefore, it would be surprising if $\sigma^{32}$ sensed the folding state of the cell by only sampling a single chaperone. Good candidates for additional regulators of $\sigma^{32}$ are GroEL/S (Hsp60/10 homologs) and HtpG (Hsp90 homolog) because, like DnaK, they are some of the most abundant chaperones in the cell, and they are highly conserved through evolution (Fink 1999). However, previous experiments with GroEL/S and HtpG have not provided evidence that they are involved in regulation of $\sigma^{32}$. First, mutations in groEL and groES that are defective in bacteriophage growth are not altered in heat shock gene regulation (Tilly et al. 1983; Straus et al. 1990). Although those mutations were assumed to be generally defective in GroEL/S function, more recent work has shown that GroEL/S has multiple classes of substrates, and mutations in groELS can have differential effects on these classes (Wang et al. 2002). It is important to note that these mutations in GroEL/S did not lead to a defect in cell growth, even though GroEL/S is essential; therefore, we now know that the lack of effect of these mutations on heat shock gene expression does not resolve this question. An additional study examining the long-term effects of GroEL/S overexpression failed to provide evidence that GroEL/S regulates $\sigma^{32}$ activity (Kanemori et al. 1994). By examining long-term GroEL overexpression, the study does not definitively rule out GroEL/S as a regulator, because it is possible that other mechanisms can compensate for long-term overexpression. Previous studies with HtpG have given evidence that it can bind to $\sigma^{32}$ in extracts, yet no experiments have been performed to determine its role in $\sigma^{32}$ regulation (Nadeau et al. 1993).

In this report, we investigate the role of these two chaperones, GroEL/S and HtpG, in regulation of the heat shock response. We present in vivo and in vitro evidence supporting the idea that GroEL/S, but not HtpG, is used to sense the protein folding state of the cell. We further show that GroEL/S is used together with the DnaK chaperone machine to regulate heat shock gene transcription, and we conclude that a cellular chaperone network regulates the activity of the $\sigma^{32}$ heat shock factor.

\section{Results}

GroEL/S and $\sigma^{32}$ show a genetic interaction

Our initial evidence that GroEL/S was a regulator of the heat shock response came from identification of a genetic interaction between $\sigma^{32}$ and GroEL/S. Overexpression of $\sigma^{32}$ is toxic, resulting in a dramatic reduction in efficiency of plating (EOP) on minimal medium. Toxicity can be alleviated by simultaneously overexpressing a negative regulator of $\sigma^{32}$ (Herman et al. 1995b). We found that overexpression of $\sigma^{32}$ alone resulted in an EOP of $1 \times 10^{-4}$, whereas simultaneous overexpression of GroEL/S and $\sigma^{32}$ restored the EOP to $\sim 1$. Therefore, GroEL/S can alleviate the toxicity of $\sigma^{32}$ overexpression, suggesting that it is a negative regulator of $\sigma^{32}$.

Overexpression of GroEL/S decreases $\sigma^{32}$-dependent transcription

We asked whether the genetic interaction between GroEL/S and $\sigma^{32}$ was due to the ability of GroEL/S to negatively regulate $\sigma^{32}$-dependent transcription. In this experiment, we compared $\sigma^{32}$-dependent transcription in cells with and without overexpression of GroEL/S, using a plasmid with the groELS operon under the control of an inducible Para promoter (Fig. 1). We assayed

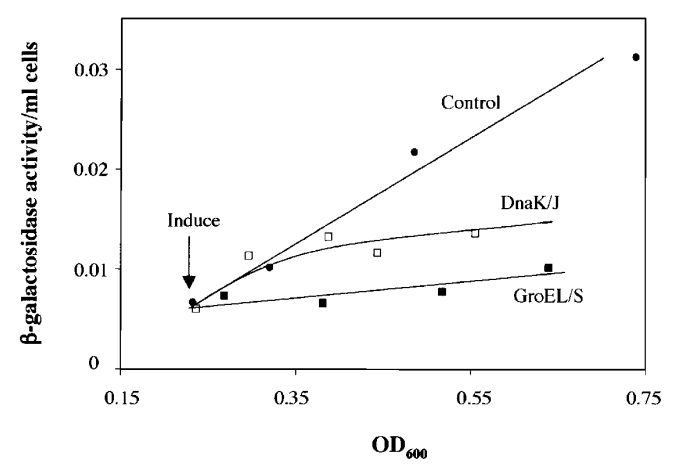

Figure 1. Effects of GroEL/S and DnaK/J overexpression on $\sigma^{32}$-dependent transcription. An exponential phase culture of strain 594 with a $\sigma^{32}$-dependent lacZ reporter and carrying either a plasmid able to overexpress GroEL/S (pGro7) or a plasmid able to overexpress DnaK/J (pKJE7) was grown at $30^{\circ} \mathrm{C}$ with and without $0.2 \%$ arabinose to induce GroEL/S or DnaK/J overexpression. A standard differential rate of synthesis plot is shown. The uninduced (control) strains gave identical results; therefore, for simplicity, we have included the data for only one control strain. This experiment and every other differential rate of synthesis experiment was performed at least three times with similar results. 
$\sigma^{32}$-dependent transcription by measuring the accumulation of $\beta$-galactosidase from a chromosomal, $\sigma^{32}$-dependent lac $Z$ transcriptional reporter as a function of cell growth. This "differential rate of $\beta$-galactosidase synthesis" measures how $\sigma^{32}$ activity changes in response to a signal over time, with the slope of the line reflecting the protein synthesis rate of $\beta$-galactosidase and therefore $\sigma^{32}$ activity. Our results indicate that GroEL/S overexpression significantly decreases $\sigma^{32}$-dependent transcription, thereby confirming the idea that GroEL/S negatively regulates $\sigma^{32}$. Next, we directly compared GroEL/S inhibition with DnaK/J inhibition. When we overexpressed DnaK/J from the same plasmid vector used for GroEL/S overexpression, we found that the eventual extent of inhibition of $\sigma^{32}$-dependent lac $Z$ reporter expression was approximately the same as that mediated by GroEL/S (Fig. 1). However, whereas GroEL/S overexpression exhibited an immediate inhibitory effect, inhibition by DnaK/J was manifest more slowly. Delayed inhibition was noticed in multiple experiments (data not shown). This effect was not due to a different extent of overexpression of the two proteins. Western blot analysis indicated that both proteins were overexpressed about 10-fold after $1 \mathrm{~h}$ of induction (data not shown). We also tested whether HtpG participated in regulation of $\sigma^{32}$. In contrast to GroEL/S and DnaK/J, overexpression of HtpG had no effect on $\sigma^{32}$-dependent lac $Z$ reporter expression (data not shown), indicating that $\mathrm{HtpG}$, at least alone, does not behave as a negative regulator of $\sigma^{32}$ in vivo.

Our results indicating that GroEL/S overexpression decreased $\sigma^{32}$ activity differed from previous results showing no effect after long-term GroEL/S overexpression (Kanemori et al. 1994), leading us to wonder whether cells can adapt to long-term GroEL/S overexpression. To determine the effects of prolonged GroEL/S overexpression, we repeated our previous experiments and re-examined the same cultures the next day. Our results confirmed that $\sigma^{32}$ activity is not repressed after long-term overexpression of GroEL/S (data not shown), indicating that the cell possesses a mechanism to adapt to long-term GroEL/S overexpression.

\section{Overexpression of GroEL decreases the activity of $\sigma^{32}$}

The experiments described earlier indicate that overexpression of GroEL/S results in decreased transcription from $\sigma^{32}$-dependent promoters in vivo. This decrease could result from a change in the amount or activity of $\sigma^{32}$, or from a combination of these two effects. To investigate this issue, we examined the synthesis rate of $\sigma^{32}$-dependent proteins, which gives us an instantaneous indication of $\sigma^{32}$ function. We also determined protein levels using Western analysis, in order to correlate $\sigma^{32}$. dependent protein synthesis with GroEL and $\sigma^{32}$ levels. We overexpressed the groELS operon on a plasmid using an inducible Ptet promoter and analyzed the synthesis of two $\sigma^{32}$-dependent proteins, HtpG and DnaK (Fig. 2A; Zhou et al. 1988). Synthesis of both HtpG and DnaK begins to decrease immediately, exhibiting a four- to
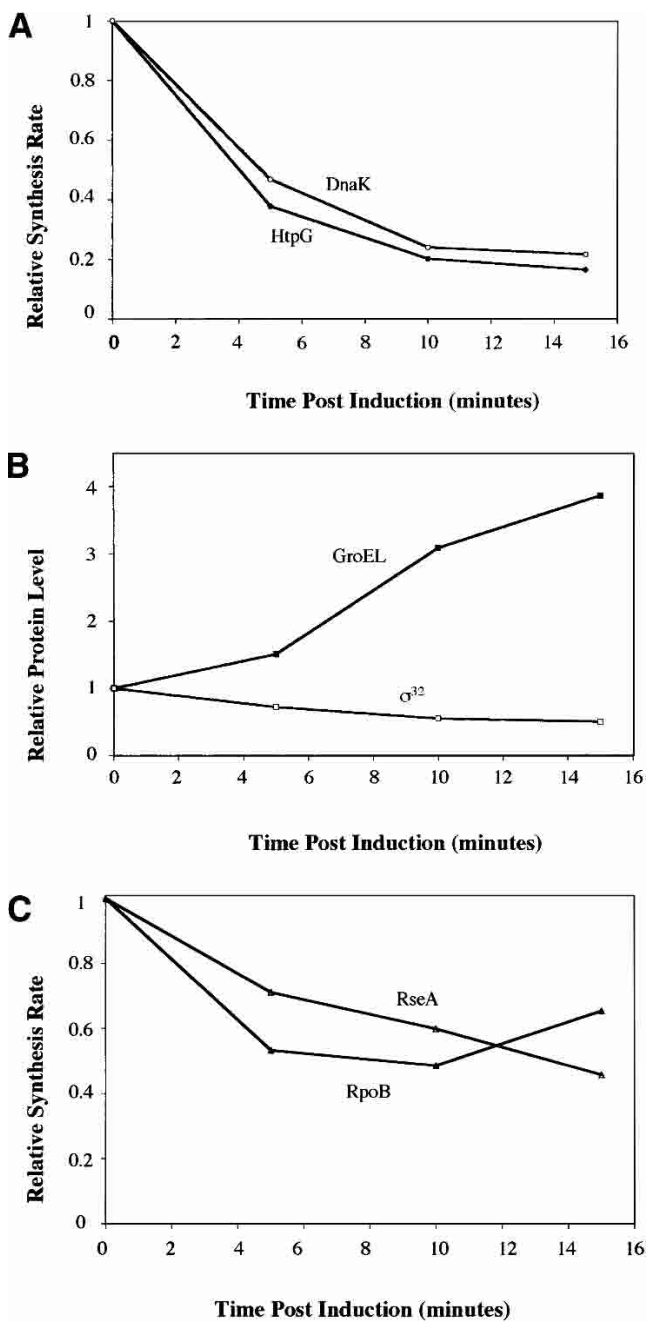

Figure 2. GroEL/S overexpression results in inhibition of $\sigma^{32}$ activity in vivo. An exponential phase culture of strain C600 carrying plasmid able to overexpress GroEL/S (pGro11) was grown in M9 minimal media containing all amino acids except for methionine and cysteine at $30^{\circ} \mathrm{C}$. Anhydrotetracycline was added at time 0 to induce GroEL/S overexpression. The rate of synthesis of two $\sigma^{32}$-dependent proteins, HtpG and DnaK $(A)$, and a $\sigma^{\mathrm{E}}$-dependent protein, RseA, and a $\sigma^{70}$-dependent protein, RpoB $(C)$, were measured. $(B)$ The level of GroEL and $\sigma^{32}$ were measured using Western analysis. All protein synthesis and Western data shown are the average from at least two independent experiments.

fivefold reduction by $10 \mathrm{~min}$ following overexpression of GroEL/S. By 10 min after GroEL/S induction, the concentration of $\sigma^{32}$ had declined less than twofold as measured by Western analysis (Fig. 2B), indicating that GroEL/S must be inhibiting the activity of $\sigma^{32}$, as the decrease in its level is insufficient to explain the drop in $\sigma^{32}$-dependent protein synthesis. To determine if these inhibitory effects occurred at physiologically relevant levels of GroEL/S, we measured GroEL levels. Western blotting revealed that the amount of GroEL increased approximately twofold at $5 \mathrm{~min}$ and fourfold over the course of our experiments (Fig. 2B). We confirmed this 


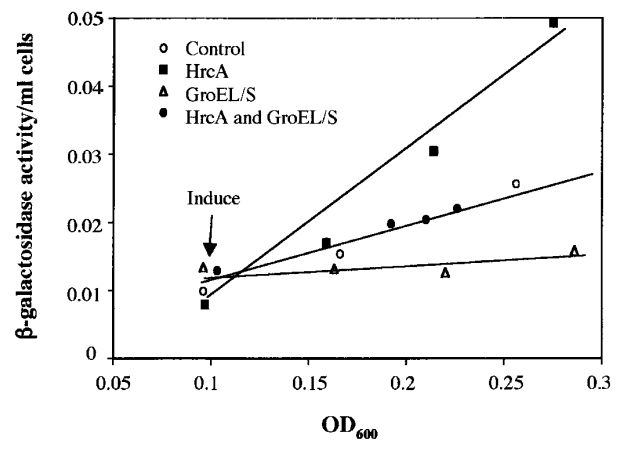

Figure 3. The ratio of GroEL/S to substrates in vivo is important for determining the activity of $\sigma^{32}$. An exponential phase culture of strain 594 carrying a plasmid able to overexpress GroEL/S (pGro7) and one able to overexpress HrcA (pJDW39) was grown in $\mathrm{LB}$ at $30^{\circ} \mathrm{C}$ in the absence of inducers (control) or in the presence of $0.2 \%$ arabinose to induce GroEL/S overexpression and/or $1 \mathrm{mM}$ IPTG to induce HrcA expression. A standard differential rate of synthesis plot is shown.

result by showing that the synthesis rate of GroEL increases $\sim 16$-fold (data not shown), which, when combined with our observed doubling time of $1 \mathrm{~h}$, allows the accumulation of GroEL to be calculated, assuming that it is a stable protein. This estimation of GroEL/S levels is in good agreement with our observed changes in GroEL with Western blotting. A two- to fourfold accumulation of GroEL/S is physiologically relevant, as cells growing at $42^{\circ} \mathrm{C}$ have two to three times as much GroEL/S as those growing at $30^{\circ} \mathrm{C}$ (Straus et al. 1990). In summary, GroEL is able to repress $\sigma^{32}$ activity with an efficiency similar to that of DnaK overexpression in vivo (Tomoyasu et al. 1998). Moreover, the negative regulation of $\sigma^{32}$ activity following GroEL/S overproduction occurs at amounts corresponding to its normal physiological variation within the cell.

As specificity controls for this experiment, we analyzed the synthesis of RseA, a $\sigma^{\mathrm{E}}$-dependent protein (De Las Penas et al. 1997), and RpoB, a $\sigma^{70}$-dependent protein (Fig. 2C; Barry et al. 1979). The synthesis of these proteins declined only twofold by $10 \mathrm{~min}$ after overexpression of GroE/S from the Ptet promoter, as compared with the four- to fivefold repression of HtpG synthesis, indicating that GroEL/S has a specific repression component for $\sigma^{32}$-mediated transcription. To ensure that the repression of $\sigma^{32}$ activity was not an artifact of the induction system, we tested two additional induction systems, Para and Plac. For each system, overexpression of GroEL/S repressed HtpG synthesis several fold more than RseA synthesis (data not shown), indicating that there is $\sigma^{32}$-specific repression of gene expression regardless of the promoter used to overexpress GroEL/S.

The ratio of GroEL/S to substrates is important for regulation of $\sigma^{32}$

We showed that increasing the levels of GroEL/S leads to down-regulation of $\sigma^{32}$ activity; however, the unfolded protein titration model suggests that it is not the total level of chaperone that is important for determining $\sigma^{32}$ activity, but the ratio of chaperone to substrate. To determine what population of GroEL/S was important for regulation of $\sigma^{32}$, we asked whether simultaneous induction of HrcA, a GroEL/S-specific substrate, would reverse the effects of GroEL/S overexpression (Mogk et al. 1997; Reischl et al. 2002; Wang et al. 2002). We found that simultaneous induction of HrcA and GroEL/S led to a reversal of the effects of GroEL/S overexpression alone (Fig. 3). This indicates that the population of GroEL/S that regulates $\sigma^{32}$ is most likely free GroEL/S not associated with substrates, confirming an important prediction of the unfolded protein titration model.

\section{Depletion of GroEL increases $\sigma^{32}$ levels}

The experiments described earlier show that overexpression of GroEL/S results in decreases in $\sigma^{32}$ activity; however, if GroEL/S is a regulator of $\sigma^{32}$, then decreases in the level of GroEL/S should lead to an increase in $\sigma^{32}$ activity. A previous report indicated that depleting GroEL/S resulted in stabilization of $\sigma^{32}$ and therefore increased $\sigma^{32}$-dependent transcription (Kanemori et al. 1994). We validated that the activity of $\sigma^{32}$ increases when GroEL/S is depleted using a strain with the chromosomal copy of groEL/S driven by the Para promoter (Fig. 4). Also, we examined whether decreasing the amount of free GroEL/S by induction of a GroEL/S-specific substrate increases $\sigma^{32}$ activity and whether this depletion also works by increasing the concentration of $\sigma^{32}$. We found that induction of HrcA increased both $\sigma^{32}$ activity and the amount of $\sigma^{32}$ (Fig. 3; data not shown). By analogy with the effects of GroEL/S depletion, this increase in amount is likely to reflect a decrease in degradation of $\sigma^{32}$. Thus, whether the concentration of free GroEL/S is decreased by depletion or titration with an

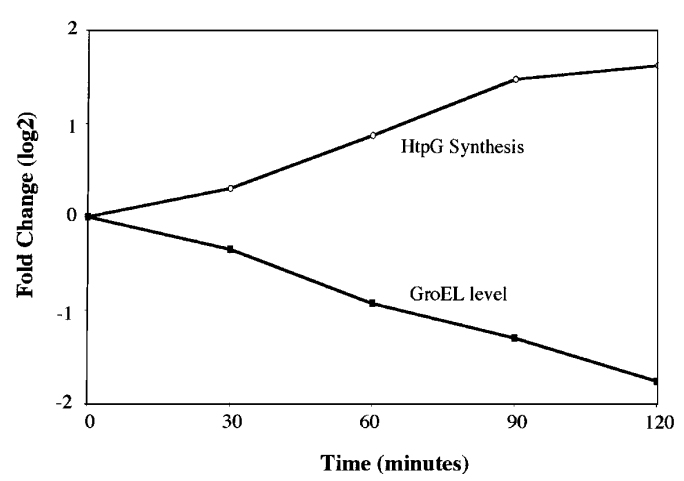

Figure 4. Depletion of GroEL/S in vivo increases $\sigma^{32}$ activity. Strain CAG48176, whose chromosomal groELS gene is driven by the inducible Para promoter, was grown in exponential phase at $30^{\circ} \mathrm{C}$ in $\mathrm{M} 9$ minimal media containing all amino acids except methionine and cysteine with $0.2 \%$ fructose as the main carbon source and $0.1 \%$ arabinose to maintain near wild-type levels of GroEL/S. Depletion of GroEL/S was initiated at time 0 by removing arabinose from the media and adding $0.2 \%$ glucose. HtpG synthesis and GroEL levels were analyzed as in Figure 2. 


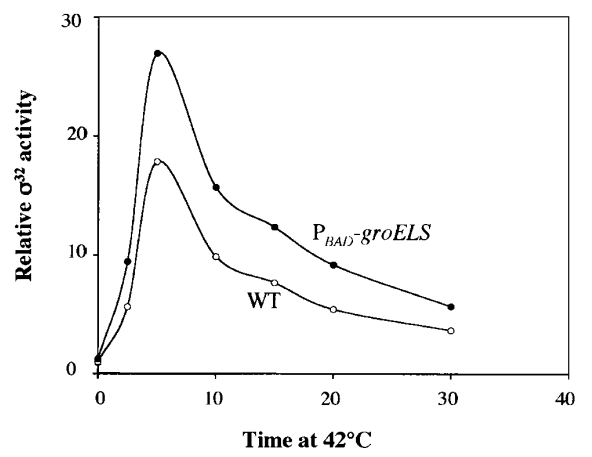

Figure 5. Changing the levels of GroEL/S increases the magnitude and duration of the heat shock response. An exponential phase culture of strain $\mathrm{C} 600$ or a derivative having the chromosomal groELS gene under control of the inducible Para promoter (CAG48176) was grown at $30^{\circ} \mathrm{C}$ and subjected to heat shock by increasing the temperature to $42^{\circ} \mathrm{C}$. $\sigma^{32}$ activity was measured by examining the rate of synthesis of HtpG.

unfolded protein substrate, the result is the same: The amount and activity of $\sigma^{32}$ increases.

\section{GroEL regulates $\sigma^{32}$ during stress conditions}

Our results with steady-state growth predict that the changes in GroEL/S levels during temperature upshift play a regulatory role in the heat shock response. We tested this idea by comparing the heat shock response of cells whose chromosomal copy of groEL/S is driven by the Para promoter with wild-type cells containing $\sigma^{32}$. mediated transcription of groE/S. Previous work established that Para groELS cells have about $80 \%$ as much GroEL as wild-type cells growing at $30^{\circ} \mathrm{C}$ (McLennan and Masters 1998). We validated that number by determining the rate of GroEL synthesis in the two strain backgrounds and showed further that after a shift to $42^{\circ} \mathrm{C}$, there is less than a twofold increase in GroEL synthesis from Para (data not shown), indicating that over the short 30 min window of a temperature upshift experiment, Para groELS cells will experience little change in GroEL level. In contrast, the wild-type cells are able to quickly increase the level of GroEL in response to a temperature upshift. We find that the heat shock response in Para groELS cells is altered in two respects (Fig. 5). First, the heat shock response is greater, showing twice as much induction at $2.5 \mathrm{~min}$ as wild-type cells, and higher peak expression at $5 \mathrm{~min}$. Second, the shut-off response is delayed compared with wild-type cells, even though all other hsps are present at higher-than-normal levels. This experiment, which has been replicated several times, indicates that even a very small $(20 \%)$ reduction in the level of GroEL prior to upshift, combined with prevention of GroEL/S accumulation during upshift, leads to a demonstrable increase in the extent and duration of the heat shock response. Together, these effects establish that GroEL/S is involved in negatively regulating heat-shock gene expression during stress conditions.

The data presented thus far argue that the level of
GroEL/S is important for $\sigma^{32}$ regulation in vivo but does not reveal whether this regulation is direct or indirect. This issue is particularly important for chaperones, which may be expected to have indirect effects. We addressed this issue in the next two sections by using in vitro experiments to determine whether GroEL/S, in the absence of other molecules, can bind to $\sigma^{32}$ and alter its transcriptional activity.

\section{GroEL binds to native $\sigma^{32}$}

We examined whether the GroEL subunit of the GroEL/S chaperone machine could bind directly to $\sigma^{32}$ in vitro using gel filtration (Fig. 6A). In this and all other in vitro experiments performed with chaperones, the chaperone preparation was first cleaned of peptides by incubation with Affi-gel blue beads (see Materials and Methods). When GroEL and $\sigma^{32}$ were incubated together and then separated on a gel filtration column, approximately onehalf of the $\sigma^{32}$ eluted in a peak coincident with free $\sigma^{32}$ and the remainder eluted in a higher molecular weight fraction, indicating binding to GroEL. This experiment
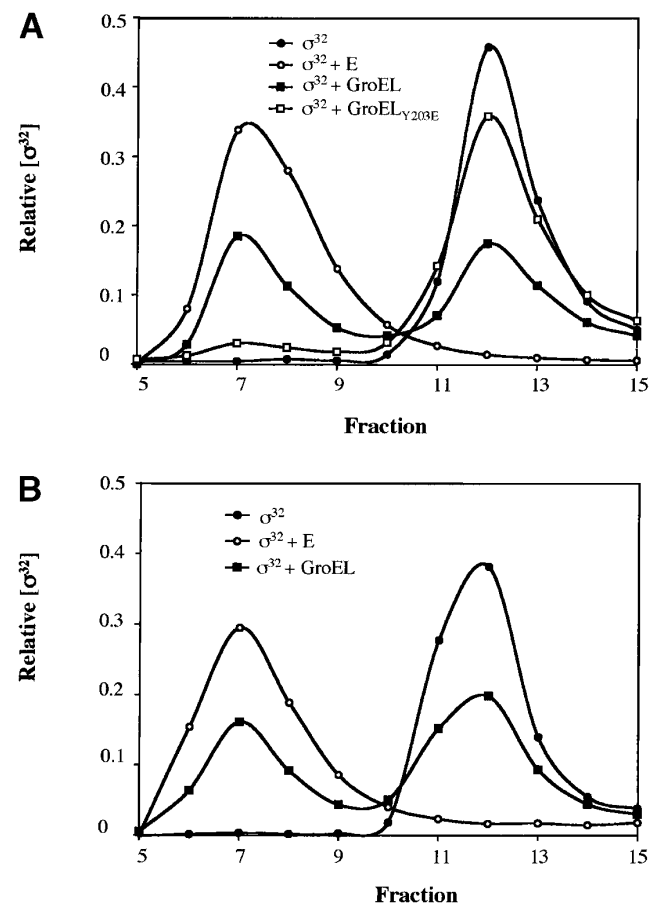

Figure 6. GroEL interacts directly with active $\sigma^{32}$ in vitro. $(A)$ Purified ${ }^{35}$ S-labeled $\sigma^{32}$ (500 nM) was incubated with GroEL (2 $\mu \mathrm{M})$, core RNAP $(2 \mu \mathrm{M})$, or a GroEL-binding mutant, GroELY203E $(2 \mu \mathrm{M})$, for $30 \mathrm{~min}$ at $20^{\circ} \mathrm{C}$ in protein-binding buffer (PBB). The proteins were then fractionated on a Superose 12 gel filtration column with $\mathrm{PBB}$ at $4^{\circ} \mathrm{C}$. Fractions were collected and counted on a scintillation counter to determine the level of $\sigma^{32}$ in each fraction. $(B)$ The free $\sigma^{32}$ peaks from the GroEL and $\sigma^{32}$-binding reaction in A were pooled and additional unlabeled $\sigma^{32}$ was added to bring the concentration to $500 \mathrm{nM}$ as in $A$. This $\sigma^{32}$ was then incubated with $2 \mu \mathrm{M}$ GroEL or $2 \mu \mathrm{M}$ core RNAP and analyzed as in $A$. 
shows that GroEL can bind directly to $\sigma^{32}$ in vitro. Two additional experiments indicated that GroEL binds to native rather than misfolded $\sigma^{32}$. First, $95 \%$ of the $\sigma^{32}$ preparation was able to bind to RNA polymerase (E), indicating that our preparation was almost completely active. Second, after our initial gel filtration with $\sigma^{32}$ and GroEL, we isolated the peak containing unbound, free $\sigma^{32}$ and reanalyzed GroEL binding (Fig. 6B). Analysis of this $\sigma^{32}$ fraction revealed that its binding characteristics were identical to those of unfractionated $\sigma^{32}$, exhibiting partial binding to GroEL and almost complete binding to RNA polymerase. This indicates that our $\sigma^{32}$ does not exist in two distinct populations, for example, one misfolded and one native, and further, that passage through the gel filtration column did not lead to inactivation or misfolding of our $\sigma^{32}$ preparation. To determine whether $\sigma^{32}$ binding to GroEL was similar to misfolded protein binding to GroEL, we tested whether a substrate-binding mutation in GroEL, GroELY203E, prevented $\sigma^{32}$ binding. This mutant is believed to affect the normal proteinbinding site of GroEL, as it is defective in the binding of several unfolded proteins in vitro and cannot complement a temperature-sensitive groEL mutant in vivo (Fenton et al. 1994). We found that GroELY203E binds very poorly to $\sigma^{32}$ in our gel filtration assay, suggesting that the normal unfolded substrate-binding site on GroEL is used for $\sigma^{32}$ binding. Taken together, these experiments show that GroEL is able to directly bind to native $\sigma^{32}$ using its normal substrate-binding site. Assuming that the binding is in equilibrium and that our GroEL preparation is mostly active, the binding constant for this reaction is $\sim 1 \mu \mathrm{M}$.

\section{The GroEL/S chaperone machine inhibits $\sigma^{32}$-dependent transcription in vitro}

GroEL binds directly to $\sigma^{32}$ in vitro, suggesting that this interaction may be sufficient to inhibit $\sigma^{32}$ function. We therefore tested whether addition of GroEL to a $\sigma^{32}$-dependent in vitro transcription reaction decreased the activity of $\sigma^{32}$. Results of a representative transcription reaction are shown in Figure 7A, and the quantified and summarized data for all experiments are shown in Figure 7B. We found that a fivefold molar excess of either GroEL or GroEL/S over $\sigma^{32}$ inhibits $\sigma^{32}$-dependent transcription in vitro approximately threefold (Fig. 7B, cf. columns 1 and 2,3, respectively). Moreover, this inhibition is specific to $\sigma^{32}$, as GroEL has no effect on $\sigma^{70}$-dependent transcription (Fig. 7B, cf. columns 7 and 8). Inhibition requires binding to $\sigma^{32}$, as the GroELY203E-binding mutant does not inhibit $\sigma^{32}$-dependent transcription (Fig. 7B, cf. columns 1 and 4). These two controls allow us to rule out that the ATPase activity of GroEL is causing the inhibition; however, we did an additional experiment to further rule out the possibility that the GroEL ATPase activity was contributing to inhibition of $\sigma^{32}$. We showed that GroEL inhibits $\sigma^{32}$ activity even when reactions are performed with $1.2 \mathrm{mM}$ ATP /six times higher than the ATP concentration normally present in the transcription reaction; data not shown). We conclude
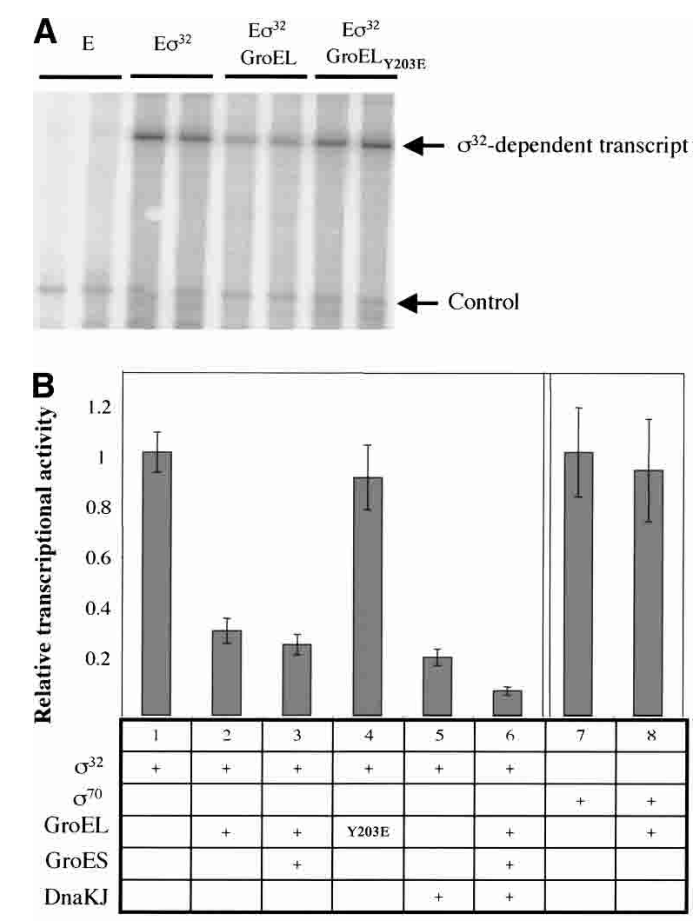

Figure 7. GroEL inhibits $\sigma^{32}$-dependent in vitro transcription. Multiround in vitro transcription was performed with holoenzyme containing either $\sigma^{32}\left(\mathrm{E}^{32}\right)$ or $\sigma^{70}\left(\mathrm{E} \sigma^{70}\right)(100 \mathrm{nM})$ incubated with GroEL (500 nM), GroES (1 $\mu \mathrm{M})$, GroELY203E (500 $n M)$, DnaK ( $2 \mu M)$, DnaJ (400 nM), or combinations thereof. An end-labeled oligo was added to each reaction as an internal control. Transcription reactions were phenol-chloroform extracted, ethanol precipitated, and analyzed on a $6 \%$ polyacrylamide gel. (A) Representative transcription gel showing duplicate reactions documenting GroEL inhibition of $E \sigma^{32}$. (B) Quantification and summary of transcription results from $A$ as well as from additional transcription experiments.

that direct binding of GroEL/S to $\sigma^{32}$ inhibits its transcription activity.

Previous work has shown that the DnaK/J chaperone machine inhibits $\sigma^{32}$ activity in an in vitro transcription reaction (Gamer et al. 1996). We replicated that result in our system using DnaK/J cleaned of peptides by passage through an Affi-gel blue column (see Materials and Methods; Fig. 7B, cf. columns 1 and 5). We then asked whether the DnaK/J and GroEL/S together further inhibited transcription. We added sufficient DnaK/J and GroEL/S to result in approximately threefold inhibition of activity by each separately and found that, together, they result in close to a ninefold decrease in $\sigma^{32}$ activity, indicating that these two chaperone machines independently inhibit $\sigma^{32}$ (Fig. 7B, cf. columns 6 and 5,3).

\section{Discussion}

A major challenge for all organisms is to maintain a constant intracellular folding environment. This requires a robust and sensitive stress response that is capable of responding to small changes in the level of misfolded 
proteins. Until now, it was believed that the DnaK chaperone machine was the sole sensor of the folding environment in E. coli. In this work, we show that every important criterion used to establish the regulatory role of DnaK also establishes a similar role for the GroEL/S chaperone machine. The use of a chaperone network to detect unfolded proteins allows the cellular folding status to be sensed more completely, and, in addition, provides a more sensitive indicator of folding state than is possible by a single chaperone machine.

GroEL/S, like DnaK, is involved in the regulation of both $\sigma^{32}$ activity and $\sigma^{32}$ stability. Overexpression of GroEL/S inhibits the activity of $\sigma^{32}$ in vivo, with efficiency approximately comparable to inhibition resulting from DnaK overexpression. In both cases, inactivation of $\sigma^{32}$ is direct, as either protein can selectively inhibit $\sigma^{32}$ transcription in vitro with comparable efficiency. There is also a link between chaperone level and the stability of $\sigma^{32}$, with underproduction of either the DnaK or GroEL/S chaperone machines stabilizing $\sigma^{32}$. However, the mechanism of this effect is unclear because it cannot be reconstituted in vitro. $\sigma^{32}$ is degraded very rapidly in vivo (T $1 / 2 \sim 1 \mathrm{~min}$ ) by the FtsH protease (Herman et al. $1995 b$; Tomoyasu et al. 1995). FtsH degrades $\sigma^{32}$ very slowly in vitro, and neither the addition of DnaK or GroEL/S separately or together facilitates proteolysis (Blaszczak et al. 1999; C. Herman, unpubl.). Recent evidence indicates that FtsH lacks a robust unfoldase activity, and an additional unidentified factor may allow FtsH to proteolyze $\sigma^{32}$ by acting as an unfoldase (Herman et al. 2003). It remains to be seen whether the chaperones work together with this factor to promote degradation, or whether they influence degradation by a more indirect pathway. Interestingly, we further show that cells can adapt to long-term overexpression of GroEL/S. This suggests a new layer of complexity in the regulation of the heat shock response. We show that, in addition to its role in the regulation of $\sigma^{32}$ under steady-state conditions, GroEL/S is important for proper regulation of $\sigma^{32}$ during stress conditions.

To our knowledge, this is the first reported example of GroEL/S binding to a folded, active protein. Moreover, our studies indicate that the normal substrate-binding site on GroEL/S mediates interaction between the two proteins. Together, these observations suggest that GroEL/S is able to bind to $\sigma^{32}$ because some aspect of the structure of native $\sigma^{32}$ mimics that of an unfolded protein. Although there are no structures of intact, uncomplexed $\sigma$ factors, a combination of high-resolution structures of individual domains and a low-resolution structure of $\sigma$ bound to RNA polymerase has led to the generally accepted notion that all $\sigma$ factors consist of domains separated by flexible linkers (Campbell et al. 2002; Murakami et al. 2002). The flexible linkers, a portion of one of the domains or the aspects of $\sigma^{32}$ that are not conserved among other $\sigma \mathrm{s}$, may be specialized to exist as an unfolded segment to allow $\sigma^{32}$ binding to GroEL/S and possibly to DnaK. In support of this idea, deuterium/hydrogen exchange followed by rapid proteolysis and mass spectrometry analysis has shown that a large portion of the $\mathrm{C}$ terminus of $\sigma^{32}$ undergoes an unusually fast exchange with the solvent, suggesting that it is either highly flexible or poorly structured (Rist et al. 2003). Such regions could serve as chaperone binding sites. As $\sigma^{32}$ is specialized to transcribe at lethal temperatures and has been shown to maintain active transcription in vitro at such temperatures (Blaszczak et al. 1995), such unstructured regions would have to exist in concert with a core folded region that allows the protein to maintain activity.

The mechanism by which binding to GroEL inhibits $\sigma^{32}$ transcriptional activity is unknown. The simplest model is that GroEL binding simply sequesters $\sigma^{32}$ in its central cavity, thereby preventing it from binding to RNA polymerase. However, the simple sequestration model seems inconsistent with our current data. First, GroEL binding to $\sigma^{32}$ appears to be much weaker than RNA polymerase binding to $\sigma^{32}$, and in the concentrations used in the in vitro transcription experiments, we would not expect GroEL to be able to compete with RNA polymerase for $\sigma^{32}$ binding. Moreover, sequestration of $\sigma^{32}$ by GroEL/S may be expected to prevent rapid $\sigma^{32}$ degradation in vivo, as GroEL normally binds proteins inside its central cavity. However, $\sigma^{32}$ does not accumulate on GroEL/S overproduction. An alternative model is that inactivation of $\sigma^{32}$ involves two processes: sequestration followed by release in a different conformation that is transiently unable to bind RNA polymerase but can still be degraded. This model may also help to explain the role of GroEL in regulation of both $\sigma^{32}$ activity and $\sigma^{32}$ stability.

The use of two independent sensors of the intracellular folding state has important consequences for the cell. First, the use of a chaperone network is expected to increase the accuracy of the surveillance for this signaltransduction pathway. Even though a single chaperone can sense a significant number of the proteins in the cell, use of both DnaK and GroEL/S allows accurate counting of the folding state for the many substrates that interact preferentially or solely with one of the two machines. In this regard, it is particularly important to sense GroEL/S occupancy, as it is the only essential chaperone and therefore folds dedicated substrates important to cellular viability. Second, the use of a chaperone network is expected to increase the sensitivity of the signal-transduction pathway. Global changes in protein folding, such as the increased misfolding during heat shock, leads to changes in protein folding for both GroEL-dependent and DnaK-dependent proteins. Sensing the levels of both classes of proteins leads to a much larger signal from a given stress, and, therefore, a much smaller change in global protein folding can be sensed.

Interestingly, there is emerging evidence in eukaryotic cells that a chaperone network may be used to regulate the heat shock response. For example, in Saccharomyces cerevisiae, the heat shock response is regulated by the transcription factor Hsfl. Hsfl has long been known to be under the control of Hsp70 (Baler et al. 1992; Halladay and Craig 1995; Shi et al. 1998), but recent evidence also implicates Hsp90 (Zou et al. 1998). This interesting par- 
allel lead us to analyze the ability of the E. coli homolog of Hsp90, HtpG, to regulate $\sigma^{32}$, but we observed no regulation in our experiments. This difference between prokaryotes and eukaryotes may be correlated with the physiology of these chaperone systems. In prokaryotes, GroEL/S is one of the most important general protein folding machines, and HtpG plays a poorly understood but presumably less critical role. In contrast, eukaryotes use Hsp90 in many important physiological pathways, whereas CCT/TriC, the eukaryotic cytoplasmic chaperonin, appears to be more specialized, although the extent of specialization is controversial (Feldman and Frydman 2000; Young et al. 2001; Hartl and Hayer-Hartl 2002). Although the two specific sensors of the protein folding environment may be different in $E$. coli and $S$. cerevisiae, it seems likely that the rationale behind having multiple sensors of protein folding has been conserved.

\section{Materials and methods}

\section{Strains}

Strains used in this study are all derivatives of K-12. All strains were isogenic with JM105, genotype supE endA sbcB15 hsdR4 rpsL thi $\Delta$ (lac-proAB) (Herman et al. 1995b); C600, genotype supE44 hsdR thi-1 thr-1 leuB6 lacY1 tonA21 (Sambrook et al. 1989); or 594, genotype lacZ-350 galK2 galT22 rpsL179

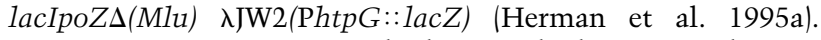
Strain CAG48176 was made by standard P1 transduction (Miller 1972) of a Para-groELS (McLennan and Masters 1998; Nielsen et al. 1999) into C600. Transductants were selected for resistance to kanamycin and confirmed by ensuring arabinosedependent growth.

\section{Media and antibiotics}

LB rich medium and M9 minimal medium were prepared as described (Sambrook et al. 1989). M9 medium was supplemented with $0.2 \%$ glucose (unless otherwise noted), $1 \mathrm{mM}$ $\mathrm{MgSO}_{4}$, and $2 \mu \mathrm{g} / \mathrm{mL}$ thiamine. Complete M9 minimal medium was also supplemented with all amino acids $(40 \mu \mathrm{g} / \mathrm{mL})$ except methionine and cysteine. When required, media was supplemented with the following antibiotics: $30 \mu \mathrm{g} / \mathrm{mL}$ kanamycin; 20 $\mu \mathrm{g} / \mathrm{mL}$ chloramphenicol; $100 \mu \mathrm{g} / \mathrm{mL}$ ampicillin, $50 \mu \mathrm{g} / \mathrm{mL}$ spectinomycin. A final concentration of $0.2 \% \mathrm{~L}-(+)$-arabinose, 25 $\mathrm{ng} / \mathrm{mL}$ anhydrotetracycline, and $1 \mathrm{mM}$ IPTG were used as inducers for Para, Ptet, Plac, and Ptac promoters.

\section{Efficiency of plating}

Overnight cultures of strain JM105 carrying either pDS1 (PtacrpoH) (Bahl et al. 1987) or pDS1 and pKV1561 (Plac-groELS) (Kanemori et al. 1994) were grown at $30^{\circ} \mathrm{C}$ in $\mathrm{M} 9$ minimal media. Serial dilutions were made and plated on M9 minimal media with and without $1 \mathrm{mM}$ IPTG at $30^{\circ} \mathrm{C}$. EOP values were calculated by dividing the number of colony forming units (cfu) in the presence of IPTG by the number of cfu in the absence of IPTG.

\section{$\beta$-Galactosidase assays}

Overnight cultures of strain 594 carrying either plasmid pGro7 (Para-groELS) (Nishihara et al. 1998), or plasmids pGro7, pJDW39 (PT5/lac-hrcA) (Wang et al. 2002), and pJM100 (lacI ${ }^{\mathrm{q}}$ ) (McCarty and Walker 1994) in LB media at $30^{\circ} \mathrm{C}$ were diluted
$1: 100$ and grown until they reached exponential phase. Cultures were then either used as a control or induced with $0.2 \%$ arabinose, $1 \mathrm{mM}$ IPTG, or both. $\sigma^{32}$ activity was assayed by monitoring $\beta$-galactosidase activity from a chromosomal $\sigma^{32}$-dependent promoter in strain 594 (Herman et al. 1995b). Samples were taken at various time points to determine $\sigma^{32}$ activity, and assays were performed as described (Miller 1972).

\section{Pulse-labeling}

For GroEL/S overexpression, saturated overnight cultures of strain C600 carrying plasmids pGro7 (Para-groELS), pKV1561 (Plac-groELS), or pGrol1 (Ptet-groELS) (Nishihara et al. 1998) grown in M9 minimal media with all amino acids except methionine and cysteine at $30^{\circ} \mathrm{C}$ were diluted $1: 100$ and then grown until they reached exponential phase. For GroEL/S depletion, an overnight culture of strain CAG48176, with the chromosomal groELS promoter replaced with Para, grown at $30^{\circ} \mathrm{C}$ in M9 minimal media containing all amino acids except methionine and cysteine and having $0.2 \%$ fructose as the carbon source and $0.1 \%$ arabinose to maintain near wild-type levels of GroEL/ $\mathrm{S}$, was diluted 1:100 and then grown until it reached exponential phase. For each time point, an $800-\mu \mathrm{L}$ aliquot of cells was pulselabeled for 1 min with EasyTag Expre ${ }^{35} S^{35} S$ protein labeling mix (NEN) followed by a chase with unlabeled methionine and cysteine. Extracts were then TCA precipitated as described in the Western blotting section. Samples were resuspended in $50 \mu \mathrm{L}$ of $2 \%$ SDS and $50 \mathrm{mM}$ Tris ( $\mathrm{pH} 7.5)$. The extracts were diluted in $750 \mu \mathrm{L}$ RIPA ( $50 \mathrm{mM}$ Tris at $\mathrm{pH} 7.5,500 \mathrm{mM} \mathrm{NaCl}, 0.1 \%$ SDS, $1 \%$ NP-40, and $0.5 \%$ sodium deoxycholate) and an aliquot was counted in a scintillation counter. To normalize the samples, we used equal numbers of counts per minute. Immunoprecipitation was done in a total volume of $750 \mu \mathrm{L}$ containing extract, polyclonal antibodies, $25 \mu \mathrm{L}$ of a 1:1 suspension of protein Aconjugated Sepharose beads, and RIPA buffer. For HtpG, DnaK, and RseA synthesis, we added an extract containing a labeled truncated version of the protein to use as an internal control prior to immunoprecipitation. The samples were rocked at $4{ }^{\circ} \mathrm{C}$ for at least $1 \mathrm{~h}$, and the beads were washed three times with 900 $\mu \mathrm{L}$ RIPA. Immunoprecipitated proteins were eluted from the beads with Laemmli sample buffer and boiling. The entire sample was then loaded onto an acrylamide gel, and the proteins were visualized using a Molecular Dynamics Storm 560 PhosphorImager scanning system.

\section{Western blotting}

Samples for Westerns $(900 \mu \mathrm{L})$ were collected and ice-cold TCA was added to a final concentration of $5 \%$. Samples were precipitated on ice for at least $30 \mathrm{~min}$, followed by centrifugation. After TCA was removed, the samples were resuspended directly in Laemmli sample buffer. An equal number of cells were loaded in each lane of the polyacrylamide gels and the proteins were transferred to nitrocellulose. The blots were probed with $1: 10,000$ dilutions of polyclonal rabbit antibodies, and then probed with 1:10,000 dilution of anti-rabbit horseradish peroxidase-conjugated secondary antibody. Western blots were developed with chemiluminescence and exposed to film. Bands were scanned and analyzed using Alpha Innotech densitometry software (Alpha Innotech).

\section{Gel filtration}

The following proteins were purified essentially as described: GroEL and GroELY203E (Fenton et al. 1994), core RNA polymerase (Sharp et al. 1999), and $\sigma^{32}$ (Gamer et al. 1996). All chaperone preparations were cleaned of misfolded proteins by incu- 
bation with Affi-Gel Blue beads overnight at $4^{\circ} \mathrm{C}$ in the presence of $5 \mathrm{mM} \mathrm{ATP}$ and $10 \mathrm{mM} \mathrm{MgCl}$. Proteins were diluted to the appropriate concentrations in protein-binding buffer $(\mathrm{PBB})$ in a final volume of $500 \mu \mathrm{L}$ and incubated at $20^{\circ} \mathrm{C}$ for at least $30 \mathrm{~min}$. PBB contains $100 \mathrm{mM} \mathrm{KCl}, 0.01 \%$ NP-40, $20 \mathrm{mM}$ Tris (pH 7.5), $5 \mathrm{mM} \mathrm{MgCl}$, and $10 \%$ glycerol. Samples were then loaded onto a Superose 12 gel filtration column and run in PBB. For $\sigma^{32}$, a fraction of the protein used was whole-cell labeled with ${ }^{35} \mathrm{~S}$ before the purification. This was added to the cold $\sigma^{32}$ before the mixture was added to the binding reaction. Thirty fractions, 1 $\mathrm{mL}$ each, were collected and the fractions were counted in a scintillation counter.

\section{In vitro transcription}

The following proteins were purified essentially as described: GroES (Fenton et al. 1994), $\sigma^{70}$ (Sharp et al. 1999), DnaK, DnaJ, and GrpE (Suh et al. 1998). All chaperone preparations were cleaned of misfolded proteins by incubation with Affi-Gel Blue beads overnight at $4^{\circ} \mathrm{C}$ in the presence of $5 \mathrm{mM}$ ATP and $10 \mathrm{mM}$ $\mathrm{MgCl}_{2}$. Holoenzyme was reconstituted by incubation of core RNA polymerase and $\sigma^{32}$ or $\sigma^{70}$ in protein dilution buffer (PDB) containing $20 \mathrm{mM}$ HEPES (pH 7.9), $100 \mathrm{mM} \mathrm{KCl}, 10 \mathrm{mM}$ $\mathrm{MgCl}_{2}, 0.1 \%$ BME, $10 \%$ glycerol, $12 \mu \mathrm{g} / \mathrm{mL}$ BSA, and $0.1 \%$ Tween. Additional proteins were added as required and samples were incubated at least $10 \mathrm{~min}$ on ice. Transcription was initiated by adding an equal volume of transcription mix containing $20 \mathrm{mM}$ HEPES (pH 7.9), $100 \mathrm{mM} \mathrm{KCl}, 10 \mathrm{mM} \mathrm{MgCl}_{2}, 0.1 \%$ BME, $50 \mathrm{nM}$ template, 2 mM ATP, 2 mM GTP, 2 mM UTP, 0.1 $\mathrm{mM}$ CTP, and $0.4 \mu \mathrm{L}{ }^{32} \mathrm{P} \alpha-\mathrm{CTP}(3000 \mathrm{Ci} / \mathrm{mmol})$, and samples were incubated $10 \mathrm{~min}$ at $30^{\circ} \mathrm{C}$. Linear DNA templates were generated using PCR, for $\mathrm{E}^{32}$ transcription, the promoter was PhtpG, and for $\mathrm{E}^{70}, \mathrm{PT} 7 A 1$ was used. Reactions were stopped by the addition of 10 volumes of transcription stop mix (TSM) containing $20 \mathrm{mM}$ EDTA, $250 \mathrm{mM} \mathrm{NaCl}, 1 \%$ SDS, and 200 $\mu \mathrm{g} / \mathrm{mL}$ glycogen. Samples were then phenol extracted to remove proteins from the reaction mixture and the RNA was ethanol precipitated and loaded onto a $6 \%$ polyacrylamide gel. As an internal control, a 60 -nucleotide, ${ }^{32} \mathrm{P}$ end-labeled oligomer was added to each reaction. The transcripts were visualized using a Molecular Dynamics Storm 560 PhosphorImager scanning system.

\section{Acknowledgments}

We thank B. Bukau for $\sigma^{32}$ antibodies, M. Masters for strain containing Para-groELS, T. Yura for plasmids, and T. Yura, K. Kim and members of the Gross lab for discussion. This work was supported by NIH Grant number GM36278, Human Frontier Program Organization Fellowship (C.H.), and NSF Graduate Research Fellowship (E.G.).

\section{References}

Arsene, F., Tomoyasu, T., and Bukau, B. 2000. The heat shock response of Escherichia coli. Int. J. Food Microbiol. 55: 3-9.

Bahl, H., Echols, H., Straus, D.B., Court, D., Crowl, R., and Georgopoulos, C.P. 1987. Induction of the heat shock response of $E$. coli through stabilization of $\sigma^{32}$ by the phage lambda cIII protein. Genes \& Dev. 1: 57-64.

Baler, R., Welch, W.J., and Voellmy, R. 1992. Heat shock gene regulation by nascent polypeptides and denatured proteins: hsp70 as a potential autoregulatory factor. I. Cell Biol. 117: 1151-1159.
Barry, G., Squires, C.L., and Squires, C. 1979. Control features within the rplJL-rpoBC transcription unit of Escherichia coli. Proc. Natl. Acad. Sci. 76: 4922-4926.

Blaszczak, A., Zylicz, M., Georgopoulos, C., and Liberek, K. 1995. Both ambient temperature and the DnaK chaperone machine modulate the heat shock response in Escherichia coli by regulating the switch between $\sigma^{70}$ and $\sigma^{32}$ factors assembled with RNA polymerase. EMBO J. 14: 5085-5093.

Blaszczak, A., Georgopoulos, C., and Liberek, K. 1999. On the mechanism of FtsH-dependent degradation of the $\sigma^{32}$ transcriptional regulator of Escherichia coli and the role of the Dnak chaperone machine. Mol. Microbiol. 31: 157-166.

Buchberger, A., Schroder, H., Hesterkamp, T., Schonfeld, H.J., and Bukau, B. 1996. Substrate shuttling between the DnaK and GroEL systems indicates a chaperone network promoting protein folding. J. Mol. Biol. 261: 328-333.

Bukau, B. 1993. Regulation of the Escherichia coli heat-shock response. Mol. Microbiol. 9: 671-680.

Bukau, B., Deuerling, E., Pfund, C., and Craig, E.A. 2000. Getting newly synthesized proteins into shape. Cell 101: 119122.

Campbell, E.A., Muzzin, O., Chlenov, M., Sun, J.L., Olson, C.A., Weinman, O., Trester-Zedlitz, M.L., and Darst, S.A. 2002. Structure of the bacterial RNA polymerase promoter specificity o subunit. Mol. Cell 9: 527-539.

Connolly, L., Yura, T., and Gross, C.A. 1999. Autoregulation of the heat shock response in procaryotes. In Molecular chaperones and folding catalysts. Regulation, cellular function and mechanism (ed. B. Bukau), pp. 13-33. Harwood Academic Publishers, Amsterdam.

Craig, E.A. and Gross, C.A. 1991. Is hsp70 the cellular thermometer? Trends Biochem. Sci. 16: 135-140.

De Las Penas, A., Connolly, L., and Gross, C.A. 1997. The $\sigma^{\mathrm{E}}$ mediated response to extracytoplasmic stress in Escherichia coli is transduced by RseA and RseB, two negative regulators of $\sigma^{\mathrm{E}}$. Mol. Microbiol. 24: 373-385.

Feldman, D.E. and Frydman, J. 2000. Protein folding in vivo: The importance of molecular chaperones. Curr. Opin. Struct. Biol. 10: 26-33.

Fenton, W.A., Kashi, Y., Furtak, K., and Horwich, A.L. 1994. Residues in chaperonin GroEL required for polypeptide binding and release. Nature 371: 614-619.

Fink, A.L. 1999. Chaperone-mediated protein folding. Physiol. Rev. 79: 425-449.

Gamer, J., Bujard, H., and Bukau, B. 1992. Physical interaction between heat shock proteins DnaK, DnaJ, and GrpE and the bacterial heat shock transcription factor $\sigma^{32}$. Cell 69: 833842.

Gamer, J., Multhaup, G., Tomoyasu, T., McCarty, J.S., Rudiger, S., Schonfeld, H.J., Schirra, C., Bujard, H., and Bukau, B. 1996. A cycle of binding and release of the DnaK, DnaJ and GrpE chaperones regulates activity of the Escherichia coli heat shock transcription factor $\sigma^{32}$. EMBO J. 15: 607-617.

Gruber, T.M. and Gross, C.A. 2003. Multiple $\sigma$ subunits and the partitioning of bacterial transcription space. Annu. Rev. Microbiol. 57: 441-466.

Halladay, J.T. and Craig, E.A. 1995. A heat shock transcription factor with reduced activity suppresses a yeast HSP70 mutant. Mol. Cell. Biol. 15: 4890-4897.

Hartl, F.U. 1996. Molecular chaperones in cellular protein folding. Nature 381: 571-579.

Hartl, F.U. and Hayer-Hartl, M. 2002. Molecular chaperones in the cytosol: From nascent chain to folded protein. Science 295: 1852-1858.

Herman, C. and Gross, C.A. 2000. Heat stress. In Encyclopedia of microbiology (ed. J. Lederberg), pp. 598-606. Academic 
Press.

Herman, C., Lecat, S., D'Ari, R., and Bouloc, P. 1995a. Regulation of the heat-shock response depends on divalent metal ions in an hflB mutant of Escherichia coli. Mol. Microbiol. 18: $247-255$.

Herman, C., Thevenet, D., D'Ari, R., and Bouloc, P. 1995b. Degradation of $\sigma^{32}$, the heat shock regulator in Escherichia coli, is governed by HflB. Proc. Natl. Acad. Sci. 92: 3516-3520.

Herman, C., Prakash, S., Lu, C.Z., Matouschek, A., and Gross, C.A. 2003. Lack of a robust unfoldase activity confers a unique level of substrate specificity to the universal AAA protease FtsH. Mol. Cell 11: 659-669.

Horwich, A.L., Weber-Ban, E.U., and Finley, D. 1999. Chaperone rings in protein folding and degradation. Proc. Natl. Acad. Sci. 96: 11033-11040.

Kanemori, M., Mori, H., and Yura, T. 1994. Effects of reduced levels of GroE chaperones on protein metabolism: Enhanced synthesis of heat shock proteins during steady-state growth of Escherichia coli. J. Bacteriol. 176: 4235-4242.

Liberek, K., Galitski, T.P., Zylicz, M., and Georgopoulos, C. 1992. The DnaK chaperone modulates the heat shock response of Escherichia coli by binding to the $\sigma^{32}$ transcription factor. Proc. Natl. Acad. Sci. 89: 3516-3520.

McCarty, J.S. and Walker, G.C. 1994. DnaK mutants defective in ATPase activity are defective in negative regulation of the heat shock response: Expression of mutant DnaK proteins results in filamentation. J. Bacteriol. 176: 764-780.

McLennan, N. and Masters, M. 1998. GroE is vital for cell-wall synthesis. Nature 392: 139.

Miller, J.H. 1972. Experiments in molecular genetics. Cold Spring Harbor Laboratory Press, Cold Spring Harbor, NY.

Mogk, A., Homuth, G., Scholz, C., Kim, L., Schmid, F.X., and Schumann, W. 1997. The GroE chaperonin machine is a major modulator of the CIRCE heat shock regulon of Bacillus subtilis. EMBO I. 16: 4579-4590.

Morimoto, R.I. 1998. Regulation of the heat shock transcriptional response: Cross talk between a family of heat shock factors, molecular chaperones, and negative regulators. Genes \& Dev. 12: 3788-3796.

Morita, M., Kanemori, M., Yanagi, H., and Yura, T. 1999a. Heatinduced synthesis of $\sigma^{32}$ in Escherichia coli: Structural and functional dissection of $\mathrm{rpoH}$ mRNA secondary structure. J. Bacteriol. 181: 401-410.

Morita, M.T., Tanaka, Y., Kodama, T.S., Kyogoku, Y., Yanagi, H., and Yura, T. 1999b. Translational induction of heat shock transcription factor $\sigma^{32}$ : Evidence for a built-in RNA thermosensor. Genes \& Dev. 13: 655-665.

Murakami, K.S., Masuda, S., and Darst, S.A. 2002. Structural basis of transcription initiation: RNA polymerase holoenzyme at $4 \AA$ resolution. Science 296: 1280-1284.

Nadeau, K., Das, A., and Walsh, C.T. 1993. Hsp90 chaperonins possess ATPase activity and bind heat shock transcription factors and peptidyl prolyl isomerases. I. Biol. Chem. 268: $1479-1487$.

Nielsen, K.L., McLennan, N., Masters, M., and Cowan, N.J. 1999. A single-ring mitochondrial chaperonin (Hsp60Hsp10) can substitute for GroEL-GroES in vivo. J. Bacteriol. 181: 5871-5875.

Nishihara, K., Kanemori, M., Kitagawa, M., Yanagi, H., and Yura, T. 1998. Chaperone coexpression plasmids: Differential and synergistic roles of DnaK-DnaJ-GrpE and GroELGroES in assisting folding of an allergen of Japanese cedar pollen, Cryj2, in Escherichia coli. Appl. Environ. Microbiol. 64: 1694-1699.

Nollen, E.A. and Morimoto, R.I. 2002. Chaperoning signaling pathways: Molecular chaperones as stress-sensing 'heat shock' proteins. J. Cell Sci. 115: 2809-2816.

Reischl, S., Wiegert, T., and Schumann, W. 2002. Isolation and analysis of mutant alleles of the Bacillus subtilis HrcA repressor with reduced dependency on GroE function. I. Biol. Chem. 277: 32659-32667.

Rist, W., Jorgensen, T.J., Roepstorff, P., Bukau, B., and Mayer, M.P. 2003. Mapping temperature-induced conformational changes in the Escherichia coli heat shock transcription factor $\sigma^{32}$ by amide hydrogen exchange. J. Biol. Chem. 278: 51415-51421.

Sambrook, J., Fritsch, E., and Maniatis, T. 1989. Molecular cloning: A laboratory manual. Cold Spring Harbor Laboratory Press, Cold Spring Harbor, NY.

Sharp, M.M., Chan, C.L., Lu, C.Z., Marr, M.T., Nechaev, S., Merritt, E.W., Severinov, K., Roberts, J.W., and Gross, C.A. 1999. The interface of $\sigma$ with core RNA polymerase is extensive, conserved, and functionally specialized. Genes \& Dev. 13: 3015-3026.

Shi, Y., Mosser, D.D., and Morimoto, R.I. 1998. Molecular chaperones as HSF1-specific transcriptional repressors. Genes \& Dev. 12: 654-666.

Straus, D.B., Walter, W.A., and Gross, C.A. 1987. The heat shock response of $E$. coli is regulated by changes in the concentration of $\sigma^{32}$. Nature 329: 348-351.

. 1989. The activity of $\sigma^{32}$ is reduced under conditions of excess heat shock protein production in Escherichia coli. Genes \& Dev. 3: 2003-2010.

-1990. DnaK, DnaJ, and GrpE heat shock proteins negatively regulate heat shock gene expression by controlling the synthesis and stability of $\sigma^{32}$. Genes \& Dev. 4: 2202-2209.

Suh, W.C., Burkholder, W.F., Lu, C.Z., Zhao, X., Gottesman, M.E., and Gross, C.A. 1998. Interaction of the Hsp70 molecular chaperone, DnaK, with its cochaperone DnaJ. Proc. Nat1. Acad. Sci. 95: 15223-15228.

Tilly, K., McKittrick, N., Zylicz, M., and Georgopoulos, C. 1983. The dnaK protein modulates the heat-shock response of Escherichia coli. Cell 34: 641-646.

Tilly, K., Spence, J., and Georgopoulos, C. 1989. Modulation of stability of the Escherichia coli heat shock regulatory factor б. J. Bacteriol. 171: 1585-1589.

Tomoyasu, T., Gamer, J., Bukau, B., Kanemori, M., Mori, H., Rutman, A.J., Oppenheim, A.B., Yura, T., Yamanaka, K., Niki, H., et al. 1995. Escherichia coli FtsH is a membranebound, ATP-dependent protease which degrades the heatshock transcription factor $\sigma^{32}$. EMBO I. 14: 2551-2560.

Tomoyasu, T., Ogura, T., Tatsuta, T., and Bukau, B. 1998. Levels of DnaK and DnaJ provide tight control of heat shock gene expression and protein repair in Escherichia coli. Mol. Microbiol. 30: 567-581.

Wang, J.D., Herman, C., Tipton, K.A., Gross, C.A., and Weissman, J.S. 2002. Directed evolution of substrate-optimized GroEL/S chaperonins. Cell 111: 1027-1039.

Young, J.C., Moarefi, I., and Hartl, F.U. 2001. Hsp90: A specialized but essential protein-folding tool. J. Cell Biol. 154: 267273.

Zhou, Y.N., Kusukawa, N., Erickson, J.W., Gross, C.A., and Yura, T. 1988. Isolation and characterization of Escherichia coli mutants that lack the heat shock $\sigma$ factor $\sigma^{32}$. J. Bacteriol. 170: 3640-3649.

Zou, J., Guo, Y., Guettouche, T., Smith, D.F., and Voellmy, R. 1998. Repression of heat shock transcription factor HSF1 activation by HSP90 (HSP90 complex) that forms a stresssensitive complex with HSF1. Cell 94: 471-480. 


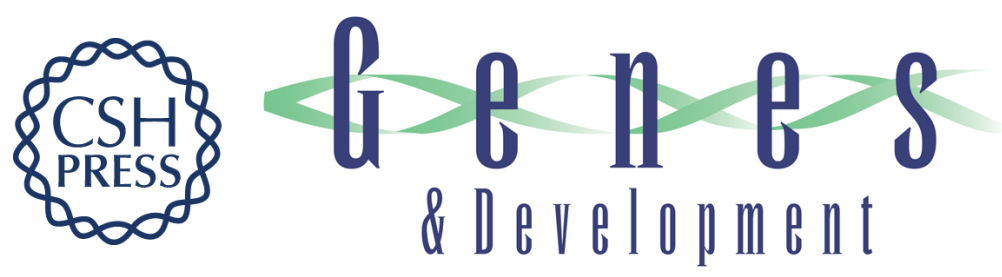

\section{A chaperone network controls the heat shock response in $E$. coli}

Eric Guisbert, Christophe Herman, Chi Zen Lu, et al.

Genes Dev. 2004, 18:

Access the most recent version at doi:10.1101/gad.1219204

References This article cites 54 articles, 28 of which can be accessed free at: http://genesdev.cshlp.org/content/18/22/2812.full.html\#ref-list-1

License

Email Alerting Service

Receive free email alerts when new articles cite this article - sign up in the box at the top right corner of the article or click here.

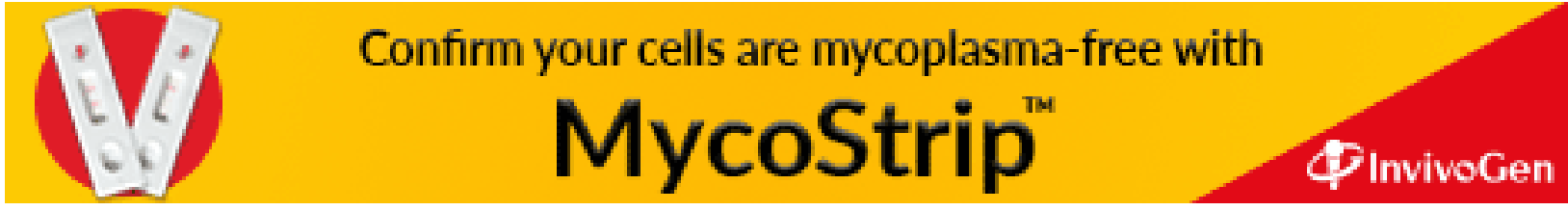

